Pontifícia Universidade $_{\text {do Rio de Janeiro }}$

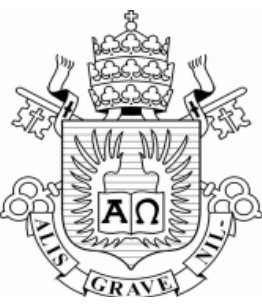

Rafaela de Gregório Dias

\title{
A seleção adversa na saída dos planos de seguro com cobertura por morte e sobrevivência
}

Dissertação apresentada como requisito parcial para obtenção do título de Mestre pelo Programa de PósGraduação em Atuária da PUC-Rio.

Orientador: Prof. Cristiano Augusto Coelho Fernandes Co-Orientador: Prof. Kaizô Iwakami Beltrão

Rio de Janeiro

Agosto de 2010 


\section{Pontifícia Universidade Católica DO RIO DE JANEIRO}

Rafaela de Gregório Dias

\section{A seleção adversa na saída dos planos de seguro com cobertura por morte e sobrevivência}

Dissertação de Mestrado

Dissertação apresentada como requisito parcial para obtenção do título de Mestre pelo Programa de PósGraduação em Atuária da PUC-Rio. Aprovada pela Comissão Examinadora abaixo assinada

Prof. Cristiano Augusto Coelho Fernandes

Orientador e Presidente

Departamento de engenharia Elétrica PUC-Rio

Prof. Kaizô Iwakami Beltrão

Co-Orientador

Escola Nacional de Ciências Estatísticas - ENCE

Prof. Mário Moreira de Carvalho de Oliveira

UFRJ

Prof. a Jéssica Quintanilha Kubrusly

UFF

Prof. ${ }^{\text {a Mônica Herz }}$

UFF

Rio de Janeiro, 23 de agosto de 2010 
Todos os direitos reservados. É proibida a reprodução total ou parcial do trabalho sem autorização da autora, do orientador e da universidade.

\section{Rafaela de Gregório Dias}

Graduou-se em Ciências Atuariais na UFMG (Universidade Federal de Minas Gerais) em 2003.

Ficha Catalográfica

Dias, Rafaela de Gregório

A seleção adversa na saída dos planos de seguro com cobertura por morte e sobrevivência / Rafaela de Gregório Dias ; orientador: Cristiano Augusto Coelho Fernandes ; co-orientador: Kaizô Iwakami Beltrão. 2010.

121 f. : il. (color.) ; $30 \mathrm{~cm}$

Dissertação (mestrado)-Pontifícia Universidade Católica do Rio de Janeiro, Instituto de Gestão de Riscos Financeiros e Atuariais, 2010.

Inclui bibliografia

1. Gestão de Riscos Financeiros e Atuariais Teses. 2. Seleção adversa. 3. Tábua de mortalidade. 4. Tábua referência mercado segurador brasileiro. 5 . Tábua seleta. I. Fernandes, Cristiano Augusto Coelho. II. Beltrão, Kaizô Iwakami. III. Pontifícia Universidade Católica do Rio de Janeiro. Instituto de Gestão de Riscos Financeiros e Atuariais. IV. Título.

CDD: 368.01 

tudo que eles representam para mim 


\section{Agradecimentos}

A Deus.

Aos meus anjos.

Ao professor Kaizô Iwakami Beltrão pela ajuda fundamental para o desenvolvimento deste trabalho. A ele minha gratidão e admiração.

Aos pesquisadores do Laboratório de Matemática Aplicada - LABMA, da UFRJ, em especial ao Ricardo Cunha Pedroso.

Ao IAPUC, à FUNENSEG e ao LABMA pelo auxílio financeiro.

Ao professor Cristiano Fernandes pelo apoio e oportunidade concedidos.

Aos meus pais, Renato e Penha, pela confiança e apoio incondicionais.

Aos meus irmãos, Pedro e Matheus pela amizade, compreensão e presença.

A todos aqueles que contribuíram de alguma forma para o meu crescimento. 


\section{Resumo}

Dias, Rafaela de Gregório; Fernandes, Cristiano Augusto Coelho. A seleção adversa na saída dos planos de seguro com cobertura por morte e sobrevivência. Rio de Janeiro, 2010. 121 p. Dissertação de Mestrado - Instituto de Gestão de Riscos Financeiros e Atuariais - IAPUC, Pontifícia Universidade Católica do Rio de Janeiro.

O estudo individualizado da mortalidade em planos de previdência e de seguros de vida é um assunto de crescente interesse tanto por parte das seguradoras quanto do órgão regulador destes segmentos, a Superintendência de Seguros Privados - SUSEP. Neste sentido, o LABMA/UFRJ calculou tábuas de referência para o mercado segurador, segregadas por sexo e cobertura, utilizando informações sobre $80 \%$ dos segurados das companhias seguradoras que atuam no Brasil. A teoria econômica prediz que existe um viés nas saídas dos planos com cobertura por sobrevivência e por morte, o que ocasiona uma diferença nas taxas de mortalidades entre o subgrupo que abandona seus planos e o grupo remanescente, e que esta diferença é variável no tempo, dependendo do número de anos decorrido desde que o indivíduo deixou o plano: assim como as tábuas seletas convergem para uma situação geral, a hipótese é a de que, passado algum tempo, a mortalidade dos indivíduos que saíram do plano converge para o mesmo nível dos que ficaram. Valendo-nos da mesma base de dados utilizada pelo LABMA/UFRJ para a construção das tábuas de referência para o mercado segurador, bem como da base de dados de registro de óbitos da previdência social, o objetivo dessa dissertação é a verificação da existência de seleção adversa nas saídas dos planos com cobertura por sobrevivência ou morte por meio da construção de tábuas seletas de mortalidade para os indivíduos que abandonam seus planos.

\section{Palavras-Chave}

Seleção Adversa; Tábua de mortalidade; Tábua de referência mercado segurador; Tábua seleta. 


\section{Abstract}

Dias, Rafaela de Gregório; Fernandes, Cristiano Augusto Coelho. Adverse selection in the output of insurance plans with coverage for death and survival. Rio de Janeiro, 2010. 121 p. Máster dissertation- Instituto de Gestão de Riscos Financeiros e Atuariais - IAPUC, Pontifícia Universidade Católica do Rio de Janeiro.

The study of mortality in individual pension plans and life insurance is a subject of growing interest both for insurers as well as for the regulator of these segments, the Superintendence of Private Insurance - SUSEP. In this sense, LABMA/UFRJ calculated mortality tables used as a reference for the insurance market, segregated by sex and coverage, using information covering $80 \%$ of the insured population originated from insurance companies operating in Brazil. Economic theory predicts that there is a bias on the outputs of plans with coverage for survival and death, which causes a difference in rates of mortality among those who quit the plan and the remaining group. This difference varies over time, depending on the time lag since the individual quit the plan: as select tables converge to the general level, the assumption here is that after some time, the mortality of individuals who left the plan converges to the same level of those who remained. Based on the same database used by LABMA/UFRJ for the construction of tables of reference for the insurance market as well as the database of deaths from the Social Security Administration, the goal of this study is to test the existence of adverse selection on the outputs of the plans with coverage for survival or death by means of constructing select mortality tables for individuals who quit their plans.

\section{Keywords}

Adverse Selection; Mortality Tables; Reference Table to the Brazilian Insurance Market; Selected Tables. 


\section{Sumário}

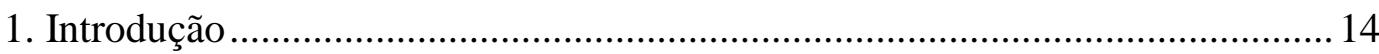

2. Teoria da informação e seleção adversa ......................................................... 19

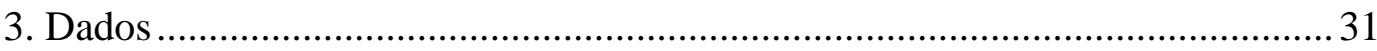

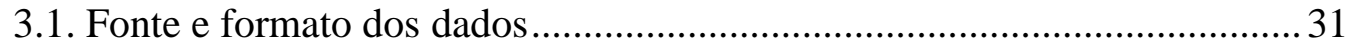

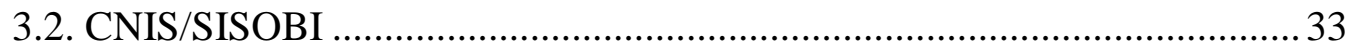

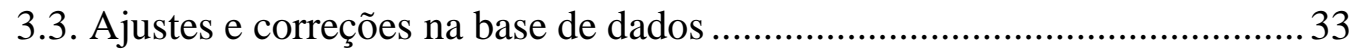

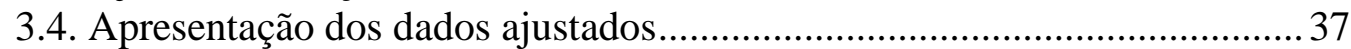

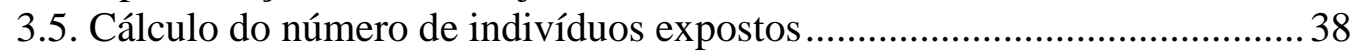

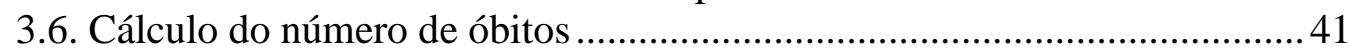

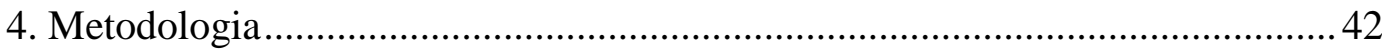

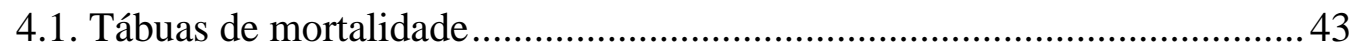

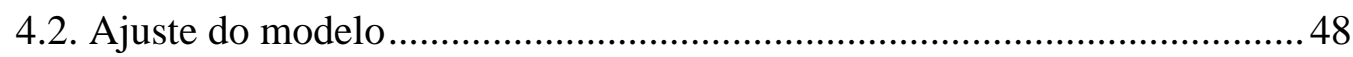

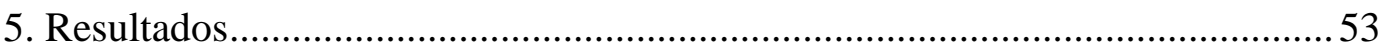

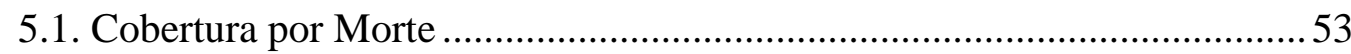

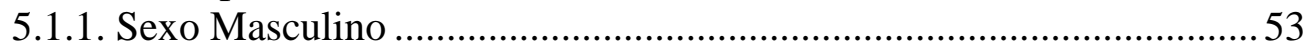

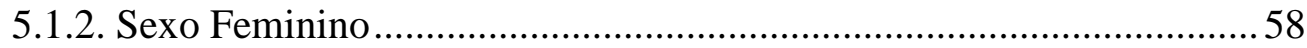

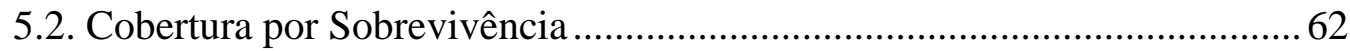

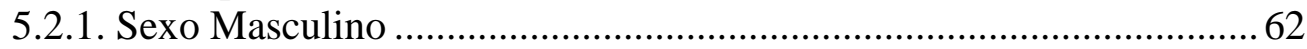

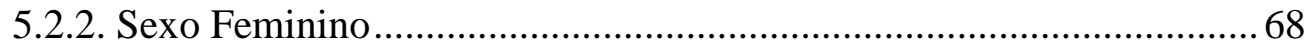

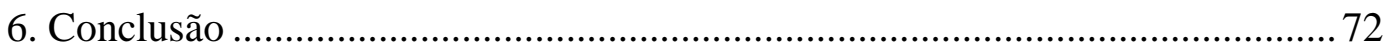

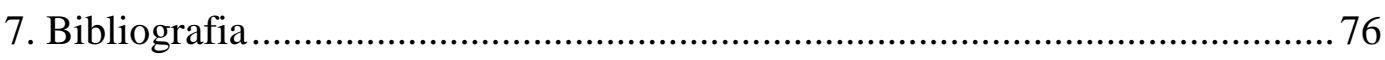

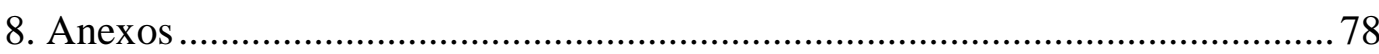




\section{Lista de tabelas}

Tabela 1 - Modelo do formato de recebimento dos dados 32

Tabela 2 - Distribuição do número de indivíduos com o CPF repetido na base de dados, para a cobertura por morte; 36

Tabela 3 - Distribuição do número de indivíduos com o CPF repetido na base de dados, para a cobertura por sobrevivência 36

Tabela 4 - Distribuição dos indivíduos na base de dados por cobertura e sexo.... 37

Tabela 5 - Exemplo de cálculo da exposição - indivíduo 1 40

Tabela 6 - Exemplo de cálculo da exposição - indivíduo 2 41

Tabela 7 - Parâmetros estimados para as razões entre as taxas específicas brutas dos dados e as da tábua BR-EMS, referentes à cobertura por morte e sexo masculino. .53

Tabela 8 - Tabela ANOVA referente à cobertura por morte e sexo masculino....54 Tabela 9- Parâmetros estimados para as razões entre as taxas específicas brutas dos dados e as da tábua BR-EMS, referentes à cobertura por morte e sexo feminino. .59

Tabela 10 - Tabela ANOVA referente à cobertura por morte e sexo feminino.... 59 Tabela 11 - Parâmetros estimados para as razões entre as taxas específicas brutas dos dados e as da tábua BR-EMS, referentes à cobertura por sobrevivência e sexo masculino. 63

Tabela 12 - Tabela ANOVA referente à cobertura por sobrevivência e sexo masculino.

Tabela 13 - Parâmetros estimados para as razões entre as taxas específicas brutas dos dados e as da tábua BR-EMS, referentes à cobertura por sobrevivência e sexo masculino. .68

Tabela 14 - Tabela ANOVA referente à cobertura por sobrevivência e sexo feminino. 


\section{Lista de Gráficos}

Gráfico 1 - Pirâmide etária para a cobertura por morte 37

Gráfico 2 - Pirâmide etária para a cobertura por sobrevivência ............................. 38

Gráfico 4 - Diagrama de Lexis para o cálculo da exposição - indivíduo 2 ........... 40

Gráfico 5 - Razão entre as taxas específicas brutas encontradas nos dados e as da tábua BR - EMS, por período de seleção, para a cobertura "Morte" e sexo "Masculino", 48

Gráfico 6- Razão entre as taxas específicas brutas encontradas nos dados e as da tábua BR - EMS, por período de seleção, para a cobertura "Morte" e sexo "Feminino". 49

Gráfico 7 - Razão entre as taxas específicas brutas encontradas nos dados e as da tábua BR - EMS, por período de seleção, para a cobertura "Sobrevivência" e sexo "Masculino", 49

Gráfico 8 - Razão entre as taxas específicas brutas encontradas nos dados e as da tábua BR - EMS, por período de seleção, para a cobertura "Sobrevivência" e sexo "Feminino". 50

Gráfico 9 - Resíduos do modelo referente à cobertura por morte e sexo masculino.

Gráfico 10 - Curva ajustada para as razões referentes à cobertura por morte e sexo masculino. 55

Gráfico 11 - Gráfico $\mathrm{q}_{\mathrm{x}}$ 's suavizados referente à cobertura por morte e sexo masculino, na escala logarítmica. .56

Gráfico 12 - Resíduos do modelo referente à cobertura por morte e sexo feminino.

Gráfico 13- Curva ajustada para as razões referentes à cobertura por morte e sexo feminino.

Gráfico 14 - Gráfico $\mathrm{q}_{\mathrm{x}}$ 's suavizados referente à cobertura por morte e sexo feminino, na escala logarítmica.

Gráfico 15 - Resíduos do modelo referente à cobertura por sobrevivência e sexo masculino.

Gráfico 16 - Curva ajustada para as razões referentes à cobertura por sobrevivência e sexo masculino .66 
Gráfico 17 - Gráfico $\mathrm{q}_{\mathrm{x}}$ 's suavizados referente à cobertura por sobrevivência e

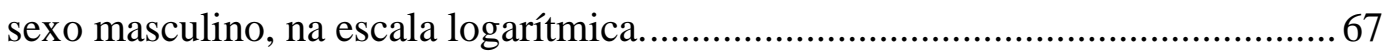

Gráfico 18 - Resíduos do modelo referente à cobertura por sobrevivência e sexo feminino 69

Gráfico 19 - Curva ajustada para as razões referentes à cobertura por sobrevivência e sexo feminino .70 Gráfico 20 - Gráfico q $\mathrm{q}_{\mathrm{x}}$ 's suavizados referente à cobertura por sobrevivência e sexo masculino, na escala logarítmica. 71 


\section{Lista de Anexos}

Anexo 1.1 - Exposição dos indivíduos referentes à cobertura por morte 80

Anexo 1.2 - Exposição dos indivíduos referentes à cobertura por sobrevivência. 83

Anexo 2.1 - Número de óbitos ocorridos referentes à cobertura por morte .86

Anexo 2.2 - Número de óbitos ocorridos referentes à cobertura por sobrevivência 89

Anexo 3.1.1 - Tábua de mortalidade para os indivíduos do sexo masculino que abandonaram o seu plano com cobertura por morte, referente ao $1^{\circ}$ período de seleção 91

Anexo 3.1.2 - Tábua de mortalidade para os indivíduos do sexo masculino que abandonaram o seu plano com cobertura por morte, referente ao $2^{\circ}$ período de seleção 93

Anexo 3.1.3 - Tábua de mortalidade para os indivíduos do sexo masculino que abandonaram o seu plano com cobertura por morte, referente ao $3^{\circ}$ período de seleção 95

Anexo 3.1.4 - Tábua de mortalidade para os indivíduos do sexo masculino que abandonaram o seu plano com cobertura por morte, referente ao $4^{\circ}$ período de seleção 97

Anexo 3.2.1- Tábua de mortalidade para os indivíduos do sexo feminino que abandonaram o seu plano com cobertura por morte, referente ao $1^{\circ}$ período de seleção .99

Anexo 3.2.2- Tábua de mortalidade para os indivíduos do sexo feminino que abandonaram o seu plano com cobertura por morte, referente ao $2^{\circ}$ período de seleção 101

Anexo 3.2.3 - Tábua de mortalidade para os indivíduos do sexo feminino que abandonaram o seu plano com cobertura por morte, referente ao $1^{\circ}$ período de seleção 103 
Anexo 3.2.4 - Tábua de mortalidade para os indivíduos do sexo feminino que abandonaram o seu plano com cobertura por morte, referente ao $4^{\circ}$ período de seleção 105

Anexo 3.3.1 - Tábua de mortalidade para os indivíduos do sexo masculino que abandonaram o seu plano com cobertura por sobrevivência, referente ao $1^{\circ}$ período de seleção 107

Anexo 3.3.2 - Tábua de mortalidade para os indivíduos do sexo masculino que abandonaram o seu plano com cobertura por sobrevivência, referente ao $2^{\circ}$ período de seleção 109

Anexo 3.3.3 Tábua de mortalidade para os indivíduos do sexo masculino que abandonaram o seu plano com cobertura por sobrevivência, referente ao $3^{\circ}$ período de seleção

Anexo 3.3.4- Tábua de mortalidade para os indivíduos do sexo masculino que abandonaram o seu plano com cobertura por sobrevivência, referente ao $4^{\circ}$ período de seleção

Anexo 3.4.1 - Tábua de mortalidade para os indivíduos do sexo feminino que abandonaram o seu plano com cobertura por sobrevivência, referente ao $1^{\circ}$ período de seleção 115

Anexo 3.4.2 - Tábua de mortalidade para os indivíduos do sexo feminino que abandonaram o seu plano com cobertura por sobrevivência, referente ao $2^{\circ}$ período de seleção

Anexo 3.4.3 - Tábua de mortalidade para os indivíduos do sexo feminino que abandonaram o seu plano com cobertura por sobrevivência, referente ao $3^{\circ}$ período de seleção 119

Anexo 3.4.4- Tábua de mortalidade para os indivíduos do sexo feminino que abandonaram o seu plano com cobertura por sobrevivência, referente ao $4^{\circ}$ período de seleção 